\title{
Femoral bone growth after extendible endoprosthetic replacement of the proximal tibia in skeletally immature patients
}

\author{
Oluwaseyi Kayode Idowu, Oladimeji Ranti Babalola, Adesegun Tibramiyu Abudu \\ From the Division of Orthopaedic Oncology, Department of Orthopaedics and Trauma, Royal Orthopaedic Hospital, \\ Birmingham, United Kingdom
}

There is increasing preference for limb salvage techniques in the management of pediatric musculoskeletal tumors. This study was aimed at evaluating femoral growth following proximal tibia resection (PTR) and placement of an extendible endoprosthesis with sliding stem. This was a retrospective study. The demographic and clinical data were collected. All the patients with malignant tumors were fully staged and commenced on appropriate chemotherapy. An expected discrepancy of $>3 \mathrm{~cm}$ was considered substantial enough to warrant extendible prosthesis. Twelve patients who had follow up full length scanogram of the lower limbs were included for the evaluation of femoral growth. The last available scanograms were used for growth comparison of the femur. The age at resection for all twelve patients ranged from 4-13 years. The commonest histological diagnosis was Osteosarcoma. In the twelve patients assessed for the growth of femur, the mean femoral length was $96 \%$ [89\%-102\%] of the unaffected femur. Distal femoral physeal growth continued after implantation of a sliding extendible prosthesis after resection of proximal tibia tumors.

Keywords : femoral bone growth; extendible endoprosthesis ; proximal tibia resection.

\section{INTRODUCTION}

Limb salvage with the use of extendible prosthesis in children with musculoskeletal tumors have become a standard procedure in the last 25 years (1). This procedure offers an acceptable alternative to amputation with a better functional result and patient survival (2). Previous studies have revealed that $50 \%$ of paediatric osteosarcoma occurs around the knee region(3). The reconstruction of proximal tibia in children presents tremendous surgical challenge because of the need to detach and reconstruct the extensor mechanism, resect the proximal tibia growth plate, violate the distal femur growth plate leading to significant growth problems $(1,4)$. Growth consideration and limb length discrepancies are pertinent in this region as the distal femoral physis

Oluwaseyi Kayode Idowu ${ }^{1}$, MBChB, MCh, FWACS, FMCS FMCOrtho,

- Oladimeji Ranti Babalola², MBBS, FWACS, FMCS, FMCOrtho,

- Adesegun Tibramiyu Abudu3, MBBS, MSc, FRCSOrth. ${ }^{1}$ Consultant Orthopaedic Surgeon, Division of Orthopaedic Oncology, Department of Orthopaedics and Trauma, National Orthopaedic Hospital, Lagos Nigeria.

${ }^{2}$ Consultant Orthopaedic Surgeon, Division of Arthroscopy and Sports Medicine, Department of Orthopaedics and Trauma, National Orthopaedic Hospital, Lagos Nigeria. ${ }^{3}$ Department of oncology, Royal Orthopaedic Hospital, Birmingham, United Kingdom.

Correspondence : Idowu Oluwaseyi Kayode, Department of Orthopaedics and Trauma, National Orthopaedic Hospital, Igbobi, Lagos, Nigeria.Tel 234.

Email : ashikoashiko@yahoo.com

- 2021, Acta Orthopædica Belgica. 
is responsible for $10 \mathrm{~mm}$ of growth per year until skeletal maturity in children (5).

Children who undergo endoprosthetic reconstruction for malignancies around the knee will usually have a sliding component inserted on the other side of the knee joint. This extendible prosthesis usually consists of a tibia component that can be lengthened, a constrained knee component and the sliding femoral component. Previous results in patients with extendible endoprosthesis after distal femur resection, suggested that the epiphysis grew on the average $63-80 \%$ of the normal side (6). However, these children often require many operations for limb lengthening, management of attendant complications, and revision to adult type of endoprosthesis (1). Complex procedures such as major orthopaedic reconstruction require both longand medium-term audit and evaluation to improve healthcare outcome (7-8).

While previous reports have evaluated the growth rate of the proximal tibia after insertion of a sliding prosthesis, a search of the available literature revealed no previous study on the critical evaluation of growth in the distal femur after insertion of a sliding prosthesis through the femur $(1,6)$. The objectives of this study was to evaluate the femoral growth following proximal tibia resection (PTR) and placement of an "extendible" endoprosthesis with sliding stem.

\section{MATERIALS AND METHODS}

This is a retrospective review from $1983-2012$ of patients who had extendible PTR at a major tertiary sarcoma center. Data were extracted from a prospectively maintained database and from the Radiology Image Archival System (PACS) for the case series. The demographic and clinical data were collected on standard proforma using Microsoft Excel spreadsheet. All the patients who had extendible PTR during the study period were included in the evaluation of the number of revision procedures. Twelve patients who had follow up full length scanogram of the lower limbs were included for the evaluation of femoral growth. The study was approved by the institution's governance unit as part of the long-term outcome study for endoprosthesis replacement in musculoskeletal oncology.

Between 1983 and 2012, ninety-six skeletally immature patients had tumour resection in the proximal tibia and extendible PTR. Patients who had full postoperative scanograms of the lower limbs were identified for growth measurements. After exclusion of patients with unclear radiographic images, twelve patients had postoperative bilateral scanograms of the lower limbs to allow for measurement and comparison of femoral length.

All the patients with malignant tumors were fully staged according to the Musculoskeletal Tumor Society Staging System and commenced on appropriate adjuvant chemotherapy according to the protocol current at the time of treatment (9). The staging investigation included plain radiographs of the lower limbs, magnetic resonance imaging (MRI) of the affected region, computerised tomography (CT) of the chest, as well as whole body bone scintigraphy.

Calibrated long-leg radiographs or scanograms of both lower limbs were also obtained as part of pre-operative evaluation for limb salvage surgery. The resection length was confirmed on coronal T2 MRI, soft tissue neo-adjuvant margin was confirmed on axial T2 MRI. An expected discrepancy of $>3 \mathrm{~cm}$ was considered substantial enough to warrant extendible prosthesis. This corresponds to approximately 13 years in children. In general, if the estimated limb length discrepancy at skeletal maturity is less than $2 \mathrm{~cm}$, an adult-type prosthesis $2 \mathrm{~cm}$ longer was inserted. This allows the operated leg to be temporarily longer but the limb usually equalises in length by skeletal maturity since the normal limb continues to grow. The implants were all designed and produced at the Department of Biomedical Engineering University College Hospital London (10,11). The Stanmore Extendible Endoprosthesis (SEER) was used in all cases. The proximal tibia endoprosthetic replacement was performed according to the previously described standard approach and rehabilitation protocols in all cases ${ }^{4}$. The operative technique included: (1) resection of the tumor, (2) reconstruction of the skeletal defect and knee joint with endoprosthesis, and (3) reconstruction of the extensor mechanism 
and soft-tissue coverage of the prosthesis with the medial gastrocnemius flaps. The incision was a long medial parapatellar incision proximal to the knee extending to the proximal one third of the leg. Thick fasciocutaneous flaps were developed to avoid skin necrosis. The popliteal trifurcation was explored early to determine if the tumor is operable. The popliteal space and trifurcation were exposed by detaching the medial gastrocnemius muscle and splitting the soleus muscle. The popliteal space was developed by identifying and detaching the origin of the medial gastrocnemius muscle and the insertion of medial hamstring muscles. The popliteal artery was identified in normal tissue and was traced distally around the popliteus muscle. The Tibiofibular joint resection was performed through the same incision. Lateral flap was developed to permit exposure of the proximal fibula. The perineal nerve was exposed and retracted prior to resection. The knee joint capsule was transected circumferentially approximately $1 \mathrm{~cm}$ away from the tibia and the patellar tendon to avoid contamination. The endoprosthesis was secured using methylmethacrylate cement into both the tibia and femur. After resection, all the patients had a medial gastrocnemius rotation flap to ensure soft tissue cover of the tibial prosthesis. The gastrocnemius flap was also incorporated into the patella tendon to reconstruct the extensor mechanism. In the postoperative period, the limb was elevated with suction drainage continued for 48 hours. Antibiotics were given at the induction of anaesthesia but not repeated. The patient began partial weight-bearing at 48 hours and was gradually allowed to increase the level of activity. Flexion to $45^{\circ}$ was permitted during the first few weeks and gentle isometric quadriceps exercises were encouraged. This was followed by regular follow up in the out-patient clinic with radiographs of the affected limb and chest.

At six weeks, patients were routinely readmitted for a week of intensive physiotherapy and hydrotherapy. They were instructed in exercises for the whole of the lower limb. By the end of this week most could walk unaided, but some required the use of a single walking stick. They were taught further exercises to enhance rehabilitation at home.

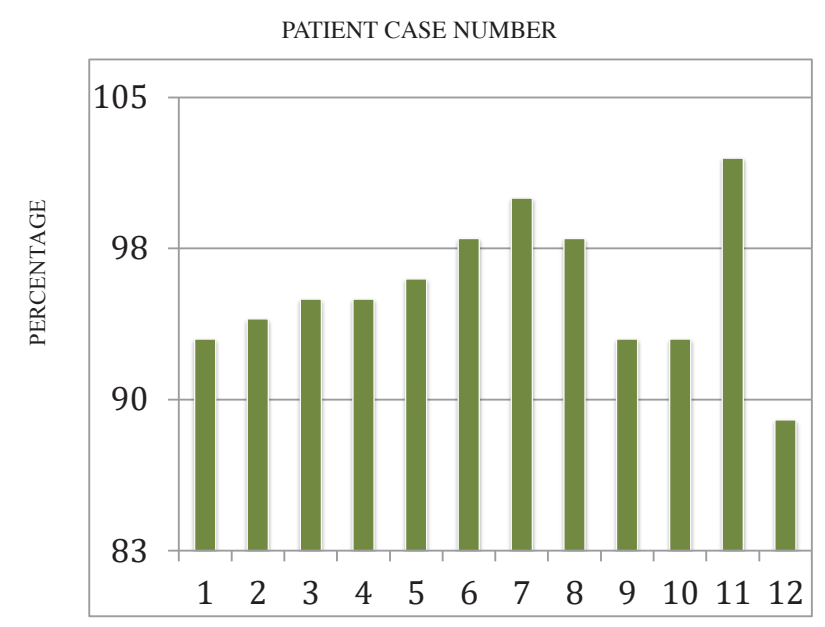

Figure 1. - Femoral length in the affected limb as a percentage of the femoral length in the unaffected limb in 12 patients.

The last available scanograms were used for the measurement and growth comparison of the femur in all the patients included. The mean period between surgery and last scanogram was 134 months (19284).

Growth measurements were performed by two observers independently and the average of the two measurements documented. The measurement was obtained between two fixed points on the femur of the salvaged limb and equivalent fixed points on the femur of the unaffected limb. The length of the femur in the affected bones was calculated as a proportion of length in the unaffected bones.

In the statistical analysis, the mean, range, and standard deviation were calculated for continuous variables using the Microsoft Excel software.

\section{RESULTS}

The age at time of surgery for all the 12 patients who underwent proximal tibia resection and sliding endoprosthesis replacement was 6-13 years (mean=10). The gender ratio was $1: 1.5$. The histological diagnoses were Osteosarcoma in 9 patients and Ewing's sarcoma in 3 patients. The Enneking stages of the lesions was predominantly stage II in 10 patients. Two patients presented with metastasis at diagnosis. The mean femoral bone length in the salvaged limb was $96 \%(89 \%-102 \%)$ of the unaffected femur (Fig. 1). There was an 
Table 1. - Patient Characteristics

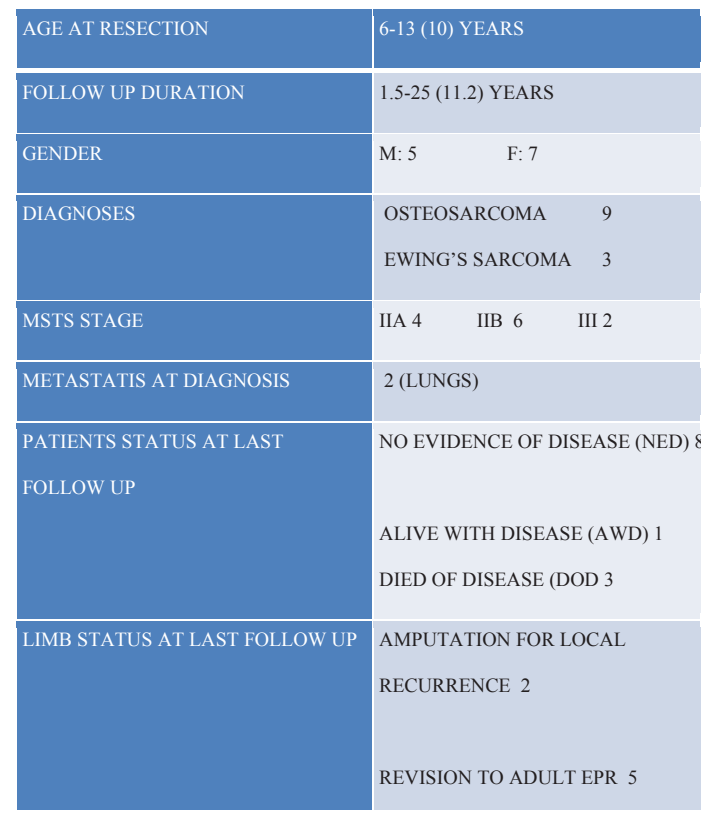

overgrowth of the salvaged femur in two patients. The mean tibia length in the salvaged limb was $94 \%$ $(59 \%-126 \%)$ of the unaffected tibia.

The patients included for growth measurement had a mean follow up of 11.2 years (range 1.525 years). Majority of the patients $($ no $=8$ ) had no evidence of the disease at the last follow up. Two patients had amputation for local recurrence to eradicate the disease, while five patients had revision to adult endoprosthesis at the last follow up (Table 1).

The length of the salvaged femur bone in the 12 children was $37-53 \mathrm{~cm}($ mean $=44 \mathrm{~cm})$, while the length of the unaffected femur bone without tumour was $28-40 \mathrm{~cm}$ (mean $=32 \mathrm{~cm})$. The mean femoral bone length in the salvaged limb was $96 \%$ [89\%$102 \%$ ] of the unaffected femur (Fig. 1). There was an overgrowth of the salvaged femur in 2 patients.

The length of the salvaged tibia bone in the 12 children was $18-42 \mathrm{~cm}($ mean $=32 \mathrm{~cm})$, while the length of the unaffected tibia bone without tumor was $26-45 \mathrm{~cm}$ (mean $=34 \mathrm{~cm})$. The mean tibia length in the salvaged tibia was $94 \%$ [59\%-126\%] of the unaffected tibia (Fig. 2).

The femoral length discrepancy in the salvaged compared to the unaffected limb was greater than two centimeters in six patients, while the tibia length

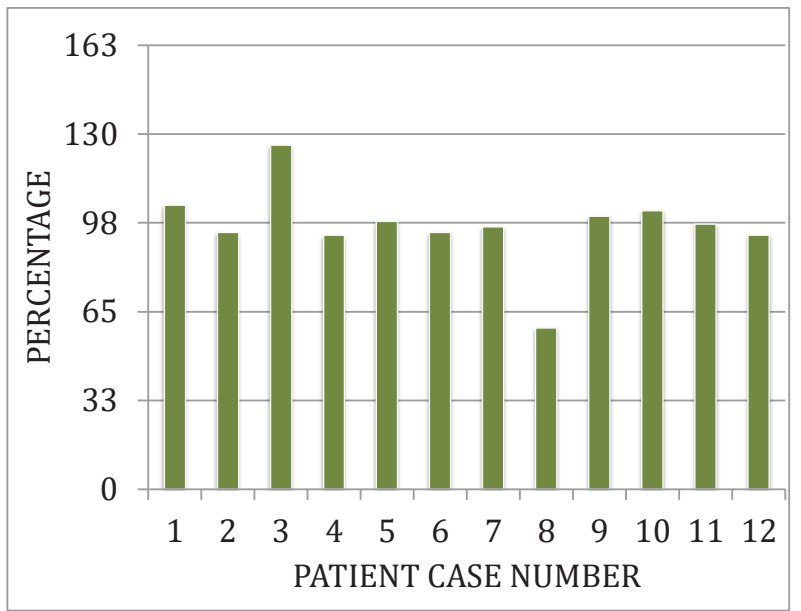

Figure 2. - Tibia length in the affected limb as a percentage of the tibia length in the unaffected limb in 12 patients.

discrepancy was greater than two centimeters in three patients. The overall limb length discrepancy in the salvaged compared to the unaffected limb was greater than two centimeters in four patients. There was overgrowth of the tibia after tumor resection in one patient (Fig. 2) and overgrowth of the affected femur in 2 patients.

\section{DISCUSSION}

Our results suggest that following extendible PTR, the use of a sliding component allowed growth in the opposite epiphysis. This is similar to previous reports on proximal tibia physeal growth following sliding endoprosthetic replacement for distal femoral tumors $(1,6)$.

While studies on proximal tibia physeal growth revealed that the affected tibia achieved $68-80 \%$ of the length of the unaffected tibia after insertion of a sliding prosthesis across the tibia, findings from this study suggests that more growth may be achievable in the distal femoral physis after the insertion of a sliding prosthesis $(89-102 \%)(1,6)$. This finding may be hinged on previous evidence that growth in the distal femoral physis is more than the proximal tibia physis by $3 \mathrm{~mm}$ per year on the average in normal patient $(12,13)$.

While the majority of the 12 patients (10 of them) had overall leg length discrepancy of greater than one centimeter, this may not affect the gait pattern of the patients clinically. The femoral 
length discrepancy in the salvaged compared to the unaffected limb was greater than two centimeters in $50 \%$ of patients, while the overall limb length discrepancy in the salvaged compared to the unaffected limb was greater than two centimeters in $33 \%$ of patients. There was overgrowth of the affected femur in 2 patients; this phenomenon has been widely reported in the traumatised femur. While the exact mechanism is not clear, it has been attributed to factors such as increased vascularity and endocrine activity following trauma or surgery around the skeletally immature femur (14-16). The overgrowth of the tibia recorded in a single patient after proximal tibia resection maybe due to the same phenomenon, or excessive lengthening of the endoprosthesis.

Knutson GA 2005 (17) evaluated data on limblength inequality obtained from radiographs, the prevalence of anatomic inequality was found to be $90 \%$, the mean magnitude of anatomic inequality was $5.2 \mathrm{~mm}$ (SD 4.1). The evidence from the previous study suggests that, for most people, anatomic leg-length inequality does not appear to be clinically significant until the magnitude reaches 2 centimeters. Moreover, JE Gordon and LE Davis 2019 (18) reported that approximately $90 \%$ of the population have a limb length discrepancy $<1.0 \mathrm{~cm}$ and there seem to be a consensus that limb length discrepancies $>2.0 \mathrm{~cm}$ are frequently a problem. In our study, contralateral epiphysiodesis was not considered an option since many patients may require revision as a result of common complications such as aseptic loosening (4).

The limitation of this study included the assumption that minimal or proportional growth occurs in the proximal femur physis. However, as this was a comparative measurement in the affected and unaffected femur of the same patient, the margin of error is likely to be minimal. Nevertheless, the measurement of growth outcome was limited to a small cohort of 12 patient with appropriate radiographs. This is largely due to the rarity of the disease condition and the complexity of this operation which is only performed in tertiary orthopaedic oncology centers. The results should be applicable to a larger group of patients with sliding endoprosthesis replacement.
Our conclusions and recommendations for practice include the fact that, it appears that distal femoral physeal growth continued after implantation of an extendible proximal endoprosthesis replacement. The study further established the advantage of limb salvage procedures in growing children and the need for long term monitoring to achieve limb equality. This is important in counselling patients and relatives at the inception of surgical treatment. In spite of the complications and the need for long term follow up, the extendible prosthesis is an effective choice in achieving equal limb length in the skeletally immature patient after resection of tumors. Further advances in implant design and biomaterials may reduce the incidence of the complications and limb length discrepancies after implantation of extendible endoprosthesis (19-20).

\section{REFERENCES}

1. Arteau A, Lewis VO, Moon BS, Satcher RL, Bird JE, Lin PP. Tibia Growth Disturbance Following Distal Femoral Resection and Expandable Endoprosthetic Reconstruction. J Bone Joint Surg Am. 2015 ; 97(22) : 1-9.

2. Finn HA, Simon MA. Limb-salvage surgery in the treatment of osteosarcoma in skeletally immature individuals. Clin Orthop Relat Res. 1991 ; 262 : 108-118.

3. Taran Shachi Jain, Taran Rakesh, Malipatil Nagraj B. (2017) Pediatric osteosarcoma : an updated review. 2017 ; 38 (1) : 33-34.

4. Grimer RJ, Belthur M, Carter SR, Tillman RM, Cool P. Extendible replacements of the proximal tibia for bone tumours. J Bone Joint Surg. 2000 ; 82-B : 255-60.

5. O'Flanagan SJ, Stack JP, McGee HM, Dervan P, Hurson. Imaging of intramedullary tumour spread in osteosarcoma. A comparison of techniques. J Bone Joint Surg Br. 1991 ; 73(6) : 998-1001.

6. Cool WP, Carter SR, Grimer RJ, Tillman RM, Walker PS. Growth after extendible endoprosthetic replacement of the distal femur. J Bone Joint Surg Br. 1997 ; 79(6) : 93842.

7. McCulloch P, Altman DG, Campbell WB, Flum DR, Glasziou P, Marshall JC. et al. No surgical innovation without evaluation : the IDEAL recommendations. Lancet. 2009 ; 374(9695) : 1105-12.

8. Paton JY, Ranmal R, Dudley J. Clinical audit : still an important tool for improving healthcare. Arch Dis Child Educ Pract Ed. $2015 ; 100(2)$ : 83-8.

9. Wolf R E, Enneking W F. The staging and surgery of musculoskeletal neoplasms. Orthop Clin North Am. 1996 ; $27: 473-481$. 
10. Scales JT, Sneath RS, Wright KWJ. Design and clinical use of extending prostheses. In : Enneking WF, ed. Limb salvage in musculoskeletal oncology. New York, etc: Churchill Livingstone. 1987 ; 52-61.

11. Unwin PS, Walker PS. Extendible endoprosthesis for the skeletally immature. Clin Orthop. 1996 ; 322 : 179-93.

12. Anderson M, Green WT, Messner MB. Growth and predictions of growth in the lower extremities. J Bone Joint Surg Am. 1963 ; 45 : 1-14.

13. Pritchett JW. Longitudinal growth and growth-plate activity in the lower extremity. Clin Orthop Relat Res. $1992 ; 275$ : 274-9.

14. Dai CQ, Yang J, Guo XS, Sun L. Risk factors for limb overgrowth after the application of titanium elastic nailing in the treatment of pediatric femoral fracture. $J$ Orthop Science 2015 ; 20(5),844-848.

15. Edvardsen P, Syversen SM. Overgrowth of the femur after fracture of the shaft in childhood. J Bone Joint Surg (Br) 1976 ; 58-B : 3, 339-342.

16. Shapiro F. Fractures of the Femoral Shaft in Children : The Overgrowth Phenomenon. Acta Orthop Scan. 1981 ; 52(6), 649-655, DOI : 10.3109/17453678108992162
17. Knutson GA. Anatomic and functional leg-length inequality: A review and recommendation for clinical decision-making. Part I, anatomic leg-length inequality : prevalence, magnitude, effects and clinical significance. Chiropr Osteopat. $2005 ; 13: 11$.

18. Gordon JE, Davis LE. Leg Length Discrepancy: The Natural History And What Do We Really Know. J Pediatr Orthop. 2019 Jul ; 39(Issue 6, Supplement 1 Suppl 1): S10-S13. doi : 10.1097/BPO.0000000000001396.

19. Gosheger G, Hardes J, Ahrens H. et al. Silver-coated megaendoprosthesis in a rabbit model--an analysis of the infection rate and toxicological side effects. Biomaterials. $2004 ; 25(24): 5547-56$.

20. Wafa H., Grimer R. J., Reddy K., Jeys L., Abudu A., Carter S. R., Tillman R. M. Retrospective evaluation of the incidence of early periprosthetic infection with silvertreated endoprosthesis in high-risk patients. Bone Joint J. 2015 ; 97-B : 2, 252-257. 